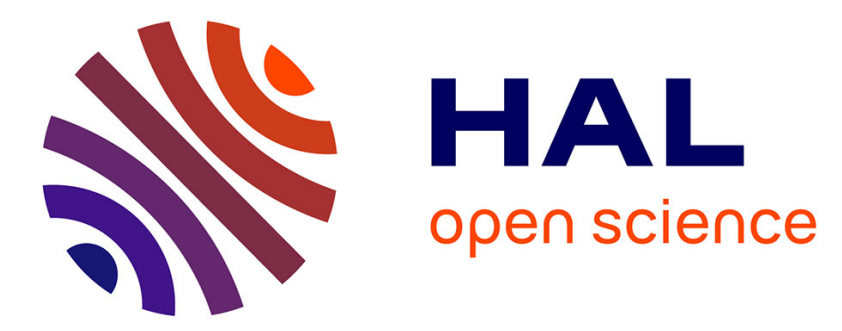

\title{
Pretreatments by means of orthogonal projections
}

\author{
J.C. Boulet, J.M. Roger
}

\section{To cite this version:}

J.C. Boulet, J.M. Roger. Pretreatments by means of orthogonal projections. Chemometrics and Intelligent Laboratory Systems, 2012, 117, p. 61 - p. 69. 10.1016/j.chemolab.2012.02.002 . hal00842452

\section{HAL Id: hal-00842452 \\ https://hal.science/hal-00842452}

Submitted on 8 Jul 2013

HAL is a multi-disciplinary open access archive for the deposit and dissemination of scientific research documents, whether they are published or not. The documents may come from teaching and research institutions in France or abroad, or from public or private research centers.
L'archive ouverte pluridisciplinaire HAL, est destinée au dépôt et à la diffusion de documents scientifiques de niveau recherche, publiés ou non, émanant des établissements d'enseignement et de recherche français ou étrangers, des laboratoires publics ou privés. 


\title{
Pretreatments by means of orthogonal projections
}

\author{
Jean-Claude Boulet ${ }^{1}$ \\ INRA, UMR1083 Sciences Pour l'Oenologie, 2 place Viala, F-34060 Montpellier, France \\ Jean-Michel Roger ${ }^{2}$ \\ IRSTEA, UMR ITAP Information Technologies Analyse environnementale Procédés \\ agricoles, F-34196 Montpellier, France
}

\begin{abstract}
This article describes several linear pretreatments based on orthogonal projections. The main differences of these pretreatments lie in the way the information to be removed is identified, using calibration dataset, pure spectra, experimental designs or mathematical models. Removing all the undesired spectral information yields spectra proportional to the net analyte signal, so it is important to collect the most complete information possible, using the complementarities of different approaches. The correction should then be processed with a single Euclidian orthogonal projection that gathers all the information, rather than with successive operations. By embedding Euclidian orthogonal projections into the calibration, it is not necessary to reapply them to new datasets.
\end{abstract}

Keywords: orthogonal projection, pretreatment, preprocessing, subspace, linear model

\section{Introduction}

Spectroscopy has spread throughout many industries as an on-line process control tool because calibration models are able to extract quantitative information about a compound of interest from the spectra. Among the models proposed, regressions or inverse calibrations such as partial least square regression (PLSR) (1), extract the relevant spectral information by means of a calibration dataset. The term "partial" recalls that just a few dimensions or latent variables are used, and thus the information related to the other variables is dropped. Pretreatments or preprocessings are positioned prior to calibration. Their purpose is to identify and to remove spectral information that interferes

\footnotetext{
Email addresses: bouletjc@supagro.inra.fr (Jean-Claude Boulet), jean-michel.roger@irstea.fr (Jean-Michel Roger)

${ }^{1}$ Tel.(33)499613148 Fax (33)499612857 (corresponding author)

${ }^{2}$ Tel.(33)467046383 Fax (33)467166440
} 
with the desired prediction. Pretreatments and regressions share a same objective, so pretreatments allow regressions to perform better. Many different pretreatments are available. This paper focuses on describing the ones based upon orthogonal projections and complements a recent review by Rinnan et al (2). After introducing the notations, we describe several pretreatments focused on orthogonal projections, and then discuss their properties. We propose a clarified view of several pretreatments by putting forward their resemblances and complementarities and suggesting the best methods for their use. The detailed relationships of these pretreatments with other pretreatments (e.g. SavitskyGolay (SG), standard normal variate) and with other calibration methods are outside the scope of this article.

\subsection{Notations}

Vectors are noted in bold lowercase, matrices in bold uppercase, and scalars in uppercase characters. Vectors are arranged in columns, except in matrices $\mathbf{X}$ and $\mathbf{X}_{G}$ where the lines represent the spectra. The transposed forms of vector $\mathbf{a}$ and matrix $\mathbf{A}$ are noted $\mathbf{a}^{\prime}$ and $\mathbf{A}^{\prime}$, respectively. The main notations are gathered in Table 1, and a glossary is also available in Table 2.

\section{Pretreatments based on orthogonal projections}

Pretreatments based on orthogonal projections deal with the correction of additive effects. For example, suppose that for sample $i$ the observed spectrum $\mathbf{x}_{i, o b s}$ is the sum of the expected spectrum $\mathbf{x}_{i}$ plus an unwanted contribution $\mathbf{h}_{i}$ :

$$
\mathbf{x}_{i, o b s}=\mathbf{x}_{i}+\mathbf{h}_{i}
$$

If a good estimation of $\mathbf{h}_{i}$ is available, the first possibility would be to perform a subtraction, and so $\mathbf{x}_{i}$ is estimated as:

$$
\widehat{\mathbf{x}}_{i}=\mathbf{x}_{i, o b s}-\widehat{\mathbf{h}}_{i}
$$

Unfortunately, because $\mathbf{h}_{i}$ is not well estimated for each spectrum $i$, this configuration is very uncommon in spectrometry. Nevertheless, it is possible to obtain a good estimation of the subspace $\mathcal{E}^{D}$ spanned by the different vectors $\left\{\mathbf{h}_{i}\right\}$. Thus it becomes possible to build a projector orthogonal to this subspace. Let $\mathbf{P}$ be a matrix of dimensions $P \times A$ whose column-vectors $\left\{\mathbf{p}_{1}, \mathbf{p}_{2}, \ldots \mathbf{p}_{A}\right\}$ form a basis of $\mathcal{E}^{D}$. Let $\mathbf{I}_{P}$ be the identity matrix of dimensions $P \times P$. The Euclidian orthogonal projector to $\mathbf{P}$ is:

$$
\mathcal{P}_{P}^{\perp}=\mathbf{I}_{P}-\mathbf{P}\left(\mathbf{P}^{\prime} \mathbf{P}\right)^{-1} \mathbf{P}^{\prime}
$$

A spectrum $\mathbf{x}_{i, \text { corr }}$ corrected from the information due to any $\mathbf{h}_{i}$ is obtained after a projection of $\mathbf{x}_{i, \text { obs }}$ orthogonally to $\mathbf{P}$ : 


$$
\mathbf{x}_{i, \text { corr }}=\mathcal{P} \stackrel{\perp}{P} \mathbf{x}_{i, o b s}=\mathcal{P} \stackrel{\perp}{P} \mathbf{x}_{i}
$$

Note that the vector $\mathbf{x}_{i, c o r r}$ that is obtained after an orthogonal projection is very different from $\widehat{\mathbf{x}}_{i}$ obtained when a subtraction is possible. However in both cases, the influence of $\mathbf{h}_{i}$ has been reduced to nought. The orthogonal projector $\mathcal{P} \frac{\perp}{P}$ is symmetrical: $\mathcal{P}_{P}^{\prime} \stackrel{\perp}{P}=\mathcal{P} \frac{\perp}{P}$, so for $N$ spectra forming the matrix $\mathrm{X}$ of dimensions $N \times P$ :

$$
\mathbf{X}_{\text {corr }}=\mathbf{X} \mathcal{P}_{P}^{\perp}=\mathbf{X}\left(\mathbf{I}_{P}-\mathbf{P}\left(\mathbf{P}^{\prime} \mathbf{P}\right)^{-1} \mathbf{P}^{\prime}\right)
$$

The performances of the different pretreatments are directly explained by their ability to obtain a good approximation of a basis of $\mathcal{E}^{D}$. Different approaches are possible: using pure spectra, information extracted from experimental design, models, and calibration datasets. For each method, matrix $\mathbf{X}$ represents centered or uncentered data, depending on the centering option chosen. In order to simplify the presentation, all pretreatments presented here are for correcting spectra in which just one compound of interest is to be quantified. However, some pretreatments can also be written for the correction of several compounds of interest.

\subsection{Pretreatment using pure spectra}

A basis of the space spanned by chemical components is given by their pure spectra. A method derived from hyperspectral imaging uses this property.

\subsubsection{Orthogonal subspace projection}

The orthogonal subspace projection (OSP) use pure spectra, called undesired signatures, which are associated with all the chemical influences present except the one of the compound of interest. These undesired signatures form matrix $\mathbf{K}$. They can be determined after a clustering process $(3 ; 4)$, in which homogeneous groups of spectra are obtained, followed by selection of a spectrum representative of each group. However in Harsanyi et al (5) they were chosen within the image. The OSP correction is a projection that is orthogonal to $\mathbf{K}$, in accordance with equation 3 :

$$
\mathbf{X}_{O S P}=\mathbf{X}\left(\mathbf{I}_{P}-\mathbf{K}\left(\mathbf{K}^{\prime} \mathbf{K}\right)^{-1} \mathbf{K}^{\prime}\right)
$$

In Harsanyi et al (5), the OSP method was applied to an hyperspectral image from an airborne VIS-IR spectrometer using the radiance spectra directly. Several end members were identified and alternatively chosen as the compound of interest. An OSP was performed for each endmember. For each OSP, the corrected spectra were used to classify the pixels, and the results were in accordance with the measured values, or ground truth. However, the limits of this method are such that all the pure spectra must be known in advance and they cannot be collinear. In addition, influences such as temperature are not taken into account. 


\subsection{Pretreatments using spectra issued from an experimental design}

If pure spectra are not available due to the chemical complexity of the samples or because the influence to be removed is physical and no pure spectrum exists, OSP cannot be applied. It is possible to construct experimental designs to obtain a matrix $\mathbf{X}_{G}$ whose spectra contain targeted spectral perturbations without any useful information. A singular value decomposition (SVD) or a principal component analysis (PCA) applied to $\mathbf{X}_{G}$ gives a matrix of eigenvectors $\mathbf{P}$ of dimensions $(P \times A)$ whose columns represent an orthonormal basis of the subspace to be removed. The matrix $\mathbf{X}$ is corrected to $\mathbf{X}_{c o r r}$ by a projection orthogonal to $\mathbf{P}$. The following formula is the same as equation 3 and can be simplified to:

$$
\mathbf{X}_{c o r r}=\mathbf{X}\left(\mathbf{I}_{P}-\mathbf{P}\left(\mathbf{P}^{\prime} \mathbf{P}\right)^{-1} \mathbf{P}^{\prime}\right)=\mathbf{X}\left(\mathbf{I}_{P}-\mathbf{P} \mathbf{P}^{\prime}\right)
$$

Several methods have been based on this principle, but they differ in the way $\mathbf{X}_{G}$ is obtained, and in how the dimension of the SVD or the PCA applied to $\mathbf{X}_{G}$ is determined.

\subsubsection{Independent interference reduction}

The independant interference reduction method (IIR), Hansen (6) uses spectra from samples where the compound of interest is null and these spectra are gathered into the matrix $\mathbf{X}_{G}$. The IIR method implies that such samples should be easy to collect in large numbers. This was the case in the reported application, due to the absence of acetone for the milk of healthy cows. IIR yielded models that were more interpretable and predictions that were more stable. IIR removes more interferences with higher numbers of PCA factors and thus the PLSR needs fewer latent variables.

\subsubsection{External parameter orthogonalization and transfer orthogonal projection}

External parameter orthogonalization (EPO), Roger et al (7) and transfer orthogonal projection (TOP), Andrew et al (8) are two closely related methods. For the same set of $M$ samples, $M$ spectra are acquired at $R$ levels of one physical influence. Thus, each sample is associated with $R$ spectra. Centering each set of $R$ spectra removes chemical information to leave only the information from the physical influence remaining. Matrix $\mathbf{X}_{G}$ of dimensions $(M R \times P)$ is obtained by merging the $R$ centered spectra from each of the $M$ samples. EPO was first applied to correct temperature effects when predicting the sugar concentration in apples in the near infrared. Ten apples were set at different temperatures between $5^{\circ} \mathrm{C}$ and $40^{\circ} \mathrm{C}$. The spectra of the ten apples were centered for each temperature level, and then merged, to yield $\mathbf{X}_{G}$. The PCA and PLSR dimensions were determined using either cross validation or Wilks $\boldsymbol{\Lambda}$. The EPO method produced a dramatic drop in the root mean square error of prediction $(R M S E P)$ value compared to that obtained without any pretreatment. TOP aims at transferring calibrations from one instrument to another, and was used in two applications: to determine the protein content in barley and moisture 
in corn. Near infrared (NIR) spectra of the same five samples of barley were acquired onto seven spectrometers, and the NIR spectra of five samples of corn were acquired onto three spectrometers. Spectra were centered for each instrument, and then merged into two matrices $\mathbf{X}_{G}$ associated with barley and corn respectively. The PCA dimensions were determined such that $98-99 \%$ of the variance was captured. For barley and corn, the TOP-PCR and TOP-PLSR models were determined on one instrument and then applied to the others. The low $R M S E P$ values produced by the analysis demonstrated the value of using TOP for calibration transfer. Calibration was also successfuly transfered to an unseen instrument (not used for the calculation of TOP).

\subsubsection{Dynamic orthogonal projection}

Dynamic orthogonal projection (DOP), Zeaiter et al (9) was inspired by EPO. The aim of DOP is the on-line correction of unexpected disruptive influences. The spectra and reference values of a few disturbed samples are assumed to be known. Let $(\mathbf{X}, \mathbf{y})$ be the calibration dataset, $\mathbf{x}_{1}$ a spectrum acquired after the appearance of a disruptive influence, and $y_{1}$ the reference value associated with $\mathbf{x}_{1}$. The ideal spectrum $\widehat{\mathbf{x}}_{1}$ which would have been obtained without the disturbance is estimated by a kernel function or weighted mean of spectra from $\mathbf{X}$ chosen for their proximity to $y_{1}$. The difference between the spectra $\left(\widehat{\mathbf{x}}_{1}\right.$ $\mathbf{x}_{1}$ ) characterizes the disturbance. The same operation is repeated with other measurement points $\mathbf{x}_{2}, \mathbf{x}_{3}$, etc., and spectral differences are gathered into $\mathbf{X}_{G}$. The method was applied to the prediction of ethanol in wine fermentations, and aimed at correcting the temperature effects. A calibration database was first obtained during an isothermal fermentation. Then, temperature was monitored during a second fermentation. Five reference points were acquired during the increase of temperature and the NIR predictions of ethanol were corrected using DOP. A Gaussian kernel function was used to build virtual spectra of the available reference points, and the database was successively corrected from one to five points. Ethanol predictions were well-corrected by DOP, even at the end of the fermentation when the temperature had decreased to its initial value. It was also shown that DOP was able to capture the vertical (baseline) and horizontal (wavelength) shifts due to the temperature.

\subsubsection{Error removal by orthogonal subtraction}

Error removal by orthogonal subtraction (EROS), Zhu et al (10) is a method derived from TOP. EROS takes into account and then corrects variations due to repetitions. Several spectra acquired from a same sample are centered. The centered spectra associated with the different samples are gathered into a matrix $\mathbf{X}_{G}$ which contains only information due to the repetitions and in which the row mean is null. EROS was applied to the diagnosis of precancerous polyps using visible-NIR spectroscopy. The repeatability of the spectra was poor due to different angles, pressures or locations at which the optical probe was used during the in vivo data acquisitions. EROS was able to correct these influences using five repetitions for each analysis. Normal and precancerous polyps were classified by principal component discriminant analysis (PCDA), the dimensions 
were tuned using cross-validation. EROS enabled the accuracy of the classification to be slightly increased. However, the models generated were expected to be more robust because they had been simplified and because EROS+PCDA needed fewer components than PCDA alone.

\subsection{Pretreatments using a polynomial model}

Let $\lambda$ be the vector of dimensions $P \times 1$ composed of values $\left\{v_{1}, v_{2}, \ldots v_{P}\right\}$. The $i^{\circ}$ column of the matrix $\boldsymbol{\Lambda}_{R}$ of dimensions $(P \times R+1)$ is obtained by raising each term of $\lambda$ to the power $i$. A vector $\mathbf{z}$ can be written in a polynomial form if it exists as vector a such that:

$$
\mathbf{z}=\Lambda_{R} \mathbf{a}
$$

If an observed spectrum $\mathbf{x}_{o b s}$ is the sum of the expected spectrum $\mathbf{x}$ plus the contribution of an unexpected influence (e.g. scattering), which is represented by a polynomial vector $\mathbf{z}$, then:

$$
\mathbf{x}_{o b s}=\mathbf{x}+\mathbf{z}=\mathbf{x}+\boldsymbol{\Lambda}_{R} \mathbf{a}
$$

An estimation of $\mathbf{x}$, corrected from the polynomial, is obtained after $\mathbf{x}$ is projected orthogonally to $\boldsymbol{\Lambda}_{R}$ :

$$
\widehat{\mathbf{x}}_{\text {corr }}=\left(\mathbf{I}_{P}-\boldsymbol{\Lambda}_{R}\left(\boldsymbol{\Lambda}_{R}^{\prime} \boldsymbol{\Lambda}_{R}\right)^{-1} \boldsymbol{\Lambda}_{R}\right) \mathbf{x}_{o b s}
$$

The following pretreatments: detrend, constrained principal spectra analysis (CPSA) and iterative polynomial fitting, are based on polynomial corrections.

\subsubsection{Detrend}

Detrend was proposed by Barnes et al $(11 ; 12)$ to correct baselin distortions due to scattering effects able to be modeled by a polynomial. These situations are very common in spectroscopy and are difficult to model due to the variability of particle sizes (2). Detrend calculates a matrix $\boldsymbol{\Lambda}_{2}$ of dimensions $(P \times 3)$ as described above, to model second-order polynomials. A detrend correction consists of an orthogonal projection of the raw spectra to $\boldsymbol{\Lambda}_{2}$. Detrend was applied to spectra from crystalline and powder forms of sucrose (11), and the corrected spectra of the same component were nearly identical. However, as expected for an orthogonal projection, they had lost the classical shape of the sucrose spectra. Other authors have proposed modeling baselines with polynomials having orders up to two $(13 ; 14)$ and with solutions for the choice of the polynomial order. However the corrections were done using subtraction and an orthogonal projection was also possible, at least for the spectra. 


\subsubsection{Constrained principal spectra analysis}

CPSA addresses the correction of influences unrelated to the chemical composition of the sample (15). As for detrend, scattering is modeled by a matrix $\boldsymbol{\Lambda}_{2}$. Spectra from external influences such as water and carbon dioxide gas, form matrix $\mathbf{K}$. Matrices $\boldsymbol{\Lambda}_{2}$ and $\mathbf{K}$ are merged to yield matrix $\mathbf{R}$. The CPSA correction consists of projecting raw spectra $\mathbf{x}_{i}$ orthogonally to $\mathbf{R}$.

\subsection{Pretreatments using data compression methods}

The main goal of data compression methods is to obtain fewer numbers of new variables $A$ such that $A<<P$, where $P$ is the dimension of a spectrum. These variables form the best basis (BB) (16), in that they span the subspace of $\mathbb{R}^{P}$ containing the most relevant information. However a consequence of this pretreatment is that information is dropped while projecting onto the BB.

Let $\mathbf{W}$ be a $P \times P$ matrix in which column-vectors constitute an orthonormal basis of $\mathbb{R}^{P}$. Thus $\mathbf{W}^{\prime} \mathbf{W}=\mathbf{I}_{P}$. The projection of any vector $\mathbf{x}_{i}$ of dimension $P$ onto $\mathbf{W}$ is invariant and gives $\mathbf{x}_{i}$ :

$$
\mathbf{W}\left(\mathbf{W}^{\prime} \mathbf{W}\right)^{-1} \mathbf{W}^{\prime} \mathbf{x}_{i}=\mathbf{W} \mathbf{W}^{\prime} \mathbf{x}_{i}=\mathbf{x}_{i}
$$

The scores of $\mathbf{x}_{i}$ in the basis represented by the column-vectors of $\mathbf{W}$ are represented by a unique vector $\mathbf{t}_{i}$, which by definition verifies:

$$
\mathbf{x}_{i}=\mathbf{W t}_{i}
$$

Thus, from equations 5 and 6 :

$$
\mathbf{t}_{i}=\mathbf{W}^{\prime} \mathbf{x}_{i}
$$

$\mathbf{W}$ is split into two matrices: $\mathbf{W}_{1: A}$ containing $A$ selected vectors of $\mathbf{W}$ (the $\mathrm{BB}$ ); and $\mathbf{W}_{A+1: P}$ containing the last $P-A$ vectors of $\mathbf{W}$. The spectrum $\mathbf{x}_{i}$ is rebuilt using $\mathbf{W}_{1: A}$ and the $A$ first scores of $\mathbf{t}_{i}$, noted $\mathbf{t}_{i, 1: A}$, into equation 6 :

$$
\mathbf{x}_{i, c o r r}=\mathbf{W}_{1: A} \mathbf{t}_{i, 1: A}=\mathbf{W}_{1: A} \mathbf{W}^{\prime}{ }_{1: A} \mathbf{x}_{i}
$$

It can easily be deduced that $\mathbf{x}_{i, c o r r}$ is also the orthogonal projection of $\mathbf{x}_{i}$ to $\mathbf{W}_{A+1: P}$ :

$$
\mathbf{x}_{i, \text { corr }}=\left(\mathbf{I}_{P}-\mathbf{W}_{A+1: P} \mathbf{W}_{A+1: P}^{\prime}\right) \mathbf{x}_{i}
$$

The equation obtained is equivalent to equation 2 with $\mathbf{W}_{A+1: P}$ replacing $\mathbf{P}$. The $\mathbf{W}_{1: A}$ and $\mathbf{W}_{A+1: P}$ matrices can be obtained using several compression methods. The simplest way is to perform a PCA, then select respectively the $A$ first and $P-A$ last components. Discrete Fourier transform (DFT) is used in signal processing. However in chemometrics more attention has been paid to wavelet transforms. Thus a discrete wavelet transform (DWT) and a wavelet packet transform (WPT) offer alternative solutions. 


\subsection{The wavelet packet transform and the discrete wavelet transform}

All of the vectors of $\mathbf{W}$ are calculated using a WPT, whereas only a few of them are obtained with DWT (16). Thus DWT is a particular case of WPT.

Two parameters must be determined for a WPT: (1) an orthonormal wavelet basis of $\mathbb{R}^{P}$, represented by the columns of $\mathbf{W}$; and (2) the $A$ vectors from $\mathbf{W}$ which form the BB (16). Examples of orthonormal wavelet families of bases are Haar-Daubechie, Symmlet, and Coiflet $(16 ; 17 ; 18)$. The vectors forming the $\mathrm{BB}$ are determined using a threshold. Several methods are available to obtain the value of the threshold: predetermined (16); minimum description length (MDL), which is a compromise between $A$ and the percent of spectral reconstruction $(16 ; 19)$; and universal thresholding (ThU) (20). Cross-validation on a calibration dataset $(\mathbf{X}, \mathbf{y})$ can help to determine these parameters $(19 ; 21)$. A WPT-DWT can be used for data compression and for smoothing, denoising and baseline corrections. Smoothing consists of removing high frequency signals $(16 ; 19 ; 22)$, regardless of their amplitudes (23). Denoising consists in removing the lower coefficients in the frequency domain, regardless of their frequencies (23). Smoothing and denoising are different, but high frequencies are often associated with low coefficients, so in practice both denoising and smoothing are performed in the same operation. Baseline can also be removed. Hu et al (20) applied DWT to Raman spectra for baseline and noise corrections.

DWT-WPT performance can be very good in terms of signal reconstruction and data compression. Barclay et al obtained corrections which overwhelmed the DFT and the SG method (23). Trygg et al obtained quite the same information after a compression rate of 30 (17). However, when associated with PLSR, DWT-WPT did not significantly improve the quality of prediction (17).

\subsection{Pretreatments using a calibration dataset}

Orthogonal signal correction (OSC) methods are based on a calibration dataset $(\mathbf{X}, \mathbf{y})$ and do not need any additional information. Several methods have been proposed for removing the spectral information orthogonal to $\mathbf{y}$ from X: Wold et al OSC (24); Sjoblom et al OSC (25); Wise et al OSC (cited in (26)); Andersson direct orthogonalization (DO) (27); Fearn's OSC (28); Westerhuis et al direct orthogonal signal correction (DOSC) (26); Goicoechea net analyte preprocessing (NAP) (29); and Trygg orthogonal projection to latent structures (OPLS) (30). These methods have been reviewed previously $(26 ; 31)$ and have been cited extensively in the litterature. OSC methods are not reviewed here but will be discussed briefly to clarify their relationships with orthogonal projections.

\subsubsection{Overview of the OSC methods}

All OSC methods aim at determining scores $\mathbf{T}$ containing information orthogonal to $\mathbf{y}$. The correction can be written as an orthogonal projection into $\mathbb{R}^{N}$ :

$$
\mathbf{X}_{O S C}=\left(\mathbf{I}_{N}-\mathbf{T}\left(\mathbf{T}^{\prime} \mathbf{T}\right)^{-1} \mathbf{T}^{\prime}\right) \mathbf{X}
$$


The methods differ in the way $\mathbf{T}$ is obtained.

- Wold's OSC

$$
\begin{aligned}
\mathbf{X}_{1: 0} & =\mathbf{X} ; \text { for step } i: \\
\mathbf{t} & =\text { first score of a PCA onto } \mathbf{X}_{1: i-1} \\
\mathbf{t}_{o} & =\left(\mathbf{I}_{N}-\mathbf{y}\left(\mathbf{y}^{\prime} \mathbf{y}\right)^{-1} \mathbf{y}^{\prime}\right) \mathbf{t} \\
\mathbf{T}_{P L S R} & =\text { first scores of a PLSR calculated with }\left(\mathbf{X}_{1: i-1}, \mathbf{t}_{o}\right) \\
\mathbf{t}_{o, \text { new }} & =\mathbf{T}_{P L S R}\left(\mathbf{T}^{\prime}{ }_{P L S R} \mathbf{T}_{P L S R}\right)^{-1} \mathbf{T}_{P L S R}^{\prime} \mathbf{t}_{0} \\
\mathbf{t} & =\mathbf{t}_{o, \text { new }} \text { then repeat until convergence of } \mathbf{t} \rightarrow \mathbf{t}_{i} \\
\mathbf{p}_{i} & =\mathbf{X}_{1: i-1}^{\prime} \mathbf{t}_{i}\left(\mathbf{t}_{i}^{\prime} \mathbf{t}_{i}\right)^{-1} \\
\mathbf{X}_{1: i} & =\left(\mathbf{I}_{N}-\mathbf{t}_{i}\left(\mathbf{t}^{\prime}{ }_{i} \mathbf{t}_{i}\right)^{-1} \mathbf{t}^{\prime}{ }_{i}\right) \mathbf{X}_{1: i-1}
\end{aligned}
$$

At each loop, the projection of $\mathbf{t}_{o}$ onto $\mathbf{T}_{P L S R}$ means that $\mathbf{t}_{o, \text { new }}$ lies in the subspace spanned by the columns of $\mathbf{X}_{1: i-1}$. Thus the $\mathbf{t}_{i}$ are orthogonal. After $A$ iterations, they form matrix $\mathbf{T}$ and the OSC correction is performed according to equation 7 .

- Sjoblom and Wise OSCs

$$
\begin{aligned}
\mathbf{X}_{1: 0} & =\mathbf{X} ; \text { for step } i: \\
\mathbf{t} & =\text { first score vector of a PCA onto } \mathbf{X}_{1: i-1} \\
\mathbf{t}_{o} & =\left(\mathbf{I}_{N}-\mathbf{y}\left(\mathbf{y}^{\prime} \mathbf{y}\right)^{-1} \mathbf{y}^{\prime}\right) \mathbf{t} \\
\mathbf{w}_{i} & =\mathbf{X}_{1: i-1}^{\prime} \mathbf{t}_{o} \text { then } \mathbf{w}_{i} \text { is normed } \\
\mathbf{t}_{1} & =\mathbf{X}_{1: i-1} \mathbf{w}_{i} \\
\mathbf{t} & =\mathbf{t}_{1} \text { then repeat until convergence of } \mathbf{t}_{1} \\
\mathbf{T}_{P L S R} & =\text { first scores of a PLSR calculated with }\left(\mathbf{X}_{1: i-1}, \mathbf{t}_{1}\right) \\
\mathbf{t}_{i} & =\mathbf{T}_{P L S R}\left(\mathbf{T}^{\prime}{ }_{P L S R} \mathbf{T}_{P L S R}\right)^{-1} \mathbf{T}_{P L S R}^{\prime} \mathbf{t}_{1} \\
\mathbf{p}_{i} & =\mathbf{X}_{1: i-1}^{\prime} \mathbf{t}_{i}\left(\mathbf{t}^{\prime}{ }_{i} \mathbf{t}_{i}\right)^{-1} \\
\mathbf{X}_{1: i} & =\left(\mathbf{I}_{N}-\mathbf{t}_{i}\left(\mathbf{t}^{\prime}{ }_{i} \mathbf{t}_{i}\right)^{-1} \mathbf{t}^{\prime}{ }_{i}\right) \mathbf{X}_{1: i-1}
\end{aligned}
$$

This is Sjoblom's OSC. Wise' OSC is similar, with an additional step consisting of orthogonalizing $\mathbf{t}_{i}$ to $\mathbf{y}(26)$. By construction each scorevector $\mathbf{t}_{i}$ lies in the $\mathbf{X}_{1: i-1}$ subspace, so the $\mathbf{t}_{i}$ vectors are orthogonal. After $A$ iterations, they form matrix $\mathbf{T}$ and the OSC correction is performed into $\mathbb{R}^{N}$ according to equation 7 .

- Andersson's DO and Goicoechea's NAP 


$$
\begin{aligned}
& \mathbf{Z}=\left(\mathbf{I}_{N}-\mathbf{y}\left(\mathbf{y}^{\prime} \mathbf{y}\right)^{-1} \mathbf{y}^{\prime}\right) \mathbf{X} \\
& \mathbf{P}=A \text { first loadings of a PCR calculated with } \mathbf{Z} \\
& \mathbf{T}=A \text { first scores of a PCR calculated with } \mathbf{Z}
\end{aligned}
$$

DO and NAP are the same method. The correction is be performed into $\mathbb{R}^{N}$ according to equation 7 , but also into $\mathbb{R}^{P}$ using $\mathbf{P}$ :

$$
\mathbf{X}_{D O-N A P}=\mathbf{X}\left(\mathbf{I}_{P}-\mathbf{P} \mathbf{P}^{\prime}\right)
$$

- Westerhuis' DOSC

$$
\begin{aligned}
\mathbf{y}_{1} & =\mathbf{X}\left(\mathbf{X}^{\prime} \mathbf{X}\right)^{+} \mathbf{X}^{\prime} \mathbf{y} \\
\mathbf{X}_{1} & =\left(\mathbf{I}_{N}-\mathbf{y}_{1}\left(\mathbf{y}^{\prime}{ }_{1} \mathbf{y}_{1}\right)^{-1} \mathbf{y}_{1}^{\prime}\right) \mathbf{X} \\
\mathbf{T} & =A \text { first scores of a PCA calculated with } \mathbf{X}_{1}
\end{aligned}
$$

As the $\mathbf{t}_{i}$ are orthogonal (28), the correction is performed into $\mathbb{R}^{N}$ according to equation 7 .

The scores of a PCA are orthogonal, so the correction is performed into $\mathbb{R}^{N}$ according to equation 7 .

- Trygg's OPLS

$$
\begin{aligned}
\mathbf{X}_{1: 0} & =\mathbf{X} ; \text { for step } i: \\
\mathbf{w}_{P L S}, \mathbf{p}_{P L S} & =\text { first weight and loading vectors of a PLSR calculated with }\left(\mathbf{X}_{1: i-1}, \mathbf{y}\right) \\
\mathbf{w}_{i} & =\mathbf{p}_{P L S}-\left(\mathbf{w}_{P L S}^{\prime} \mathbf{p}_{P L S}\right)\left(\mathbf{w}_{P L S}^{\prime} \mathbf{w}_{P L S}\right)^{-1} \mathbf{w}_{P L S} \text { then } \mathbf{w}_{i} \text { is normed } \\
\mathbf{t}_{i} & =\mathbf{X}_{1: i-1} \mathbf{w}_{i} \\
\mathbf{p}_{i} & =\mathbf{X}_{1: i-1}^{\prime} \mathbf{t}_{i}\left(\mathbf{t}_{i}^{\prime} \mathbf{t}_{i}\right)^{-1} \\
\mathbf{X}_{1: i} & =\left(\mathbf{I}_{N}-\mathbf{t}_{i}\left(\mathbf{t}^{\prime}{ }_{i} \mathbf{t}_{i}\right)^{-1} \mathbf{t}^{\prime}{ }_{i}\right) \mathbf{X}_{1: i-1}
\end{aligned}
$$

Each vector $\mathbf{t}_{i}$ lies in the column space of $\mathbf{X}_{1: i-1}$. Thus the $\mathbf{t}_{i}$ vectors are orthogonal and can be gathered into a matrix $\mathbf{T}$ for the OPLS correction into $\mathbb{R}^{N}$ according to equation 7 . 


\subsubsection{Properties of the $O S C$ methods}

OSC methods were created to improve inner properties (26), such as: no (or nearly no) correlation between the OSC scores $\mathbf{t}_{i}$ and the reference values $\mathbf{y}$; removal of the largest variability in $\mathbf{X}$; belonging of the $\mathbf{t}_{i}$ to the column space; and more direct calculation. In order to prevent overfit, just a few dimensions are usually removed. The usefulness of OSC has been widely discussed, and many authors agree that OSC does not improve accuracy $(26 ; 27 ; 28 ; 29 ; 30 ; 31)$. Several OSC methods are connected with PLSR. For example, Wold's OSC is derived from PLSR, and OPLS can be obtained from PLSR (32). Often the same calibrations obtained after OSC would be obtained without OSC, but the latter calibrations contain more PLSR latent variables. Nevertheless OSC improves the interpretability of the calibrations by identifying outliers (27) and explaining the regression model obtained after OSC, by analyzing the information removed by OSC $(30 ; 31)$.

OSC models can always be presented as a subtraction: $\mathbf{X}_{O S C}=\mathbf{X}-\sum \mathbf{t}_{i} \mathbf{p}_{i}{ }_{i}$ (31). The calculations of these models are similar to orthogonal projection into the individual space $\mathbb{R}^{N}$. It is less obvious to link OSC to orthogonal projections into the variable space $\mathbb{R}^{P}$, but it is possible in some instances. For example, DO and NAP are Euclidian orthogonal projections into $\mathbb{R}^{P}$, whereas Fearn's OSC is an oblique orthogonal projection into $\mathbb{R}^{P}$, see Appendix A. These mathematical considerations are of importance for the embedded or not embedded properties of OSCs, and also for their compatibilities with other orthogonal projection methods (see below).

\section{Discussion}

Orthogonal projections are common to the main linear pretreatment methods. We discuss several of their properties, including the relationship to the net analyte signal, the complementarity and the association of different pretreatments, and the correction of additive and multiplicative effects. We also propose for practical rules using orthogonal projections.

\subsection{Net analyte signal and pretreatments in the calibration process}

Calibration consists of two steps: an optional pretreatment or preprocessing step, and a mandatory calibration step. The aims of pretreatments and linear calibrations are linked.

A linear multivariate calibration extracts the spectral information produced by the compound of interest, and uses this information to predict the concentration of the compound. From a mathematical point of view, it is a function from $\mathbb{R}^{P}$ to $\mathbb{R}$, represented by a vector $\mathbf{b}$, called the regression or b-coefficients vector. Let $(\mathbf{X}, \mathbf{y})$ be a calibration dataset, $\left(\mathbf{X}_{c}, \mathbf{y}_{c}\right)$ be the calibration datset after mean centering, and let $\mathbf{b}$ and $\mathbf{b}_{c}$ be the models obtained from raw or centered data. For a spectrum $\mathbf{x}_{i}$ from $\mathbf{X}$ acquired on sample $i$, an estimation $\widehat{y}_{i}$ for the concentration of the compound of interest is given by the following equations, depending on the centering option: 


$$
\begin{aligned}
& \widehat{y}_{i}=\mathbf{x}_{i}^{\prime} \mathbf{b} \text { (no centering) } \\
& \widehat{y}_{i}=\mathbf{x}^{\prime}{ }_{i, c} \mathbf{b}_{c}+y_{0} \text { (centering) }
\end{aligned}
$$

The intercept $y_{0}$ is induced by the centering. A good calibration model gives a prediction close to the true values of $y_{i}$, so $\left|y_{i}-\widehat{y}_{i}\right|$ is minimized under different constraints. For that purpose, it is able to extract only the relevant information from $\mathbf{x}_{i}$ (or $\mathbf{x}_{i, c}$ if centered). The most condensed relevant spectral information is called the net analyte signal (NAS). Lorber et al (33) introduced the NAS (a vector of the same dimension as a spectrum) in multivariate calibration. For one component of interest, two definitions were proposed:

- First definition

"NAS is the part of the spectrum of the component of interest which, is orthogonal to the spectra of the other components" $(33 ; 34)$

- Second definition

"NAS is the part of the raw signal that is useful for prediction of the component of interest" (34)

Suppose that all of the contributions to the spectra, except the contribution of the compound to be predicted, span a subspace $\mathcal{E}^{D}$. Let the column-vectors of a matrix $\mathbf{P}$ be a basis of $\mathcal{E}^{D}$. Thus the NAS part of a spectrum $\mathbf{x}_{i}$, called $\mathbf{x}_{i, n a s}$, is obtained as an orthogonal projection of $\mathbf{x}_{i}$ to $\mathbf{P}(35)$, so that the NAS is in the null space of $\mathcal{E}^{D}$ :

$$
\mathbf{x}_{i, \text { nas }}=\left(\mathbf{I}_{P}-\mathbf{P}\left(\mathbf{P}^{\prime} \mathbf{P}\right)^{-1} \mathbf{P}^{\prime}\right) \mathbf{x}_{i}
$$

The net sensivity vector (NSV) $\mathbf{s}_{n s v}$ is the NAS for a compound of interest whose concentration is one. The vectors $\mathbf{s}_{n s v}$ and $\mathbf{b}$ are collinear and they verify, for direct and inverse calibrations (34):

$$
\mathbf{s}_{n s v}=\mathbf{b}\left(\mathbf{b}^{\prime} \mathbf{b}\right)^{-1}
$$

For the same compound of interest, the NAS is strongly dependent on the experiment. For instance, glucose presents different NAS, when measured in fruit juices or blood. If a new compound whose spectra is close to that of glucose is added either to juice or blood, the glucose NAS is immediately modified. Thus, the NAS is conceptual rather than actual and is difficult or impossible to measure. However, the NAS can be estimated. From equation 9, the best calibration models are expected to be the best NAS estimates.

Equations 8 and 9 also clarify the relationships among orthogonal pretreatments, NAS and calibrations. The definitions of the NAS and a pretreatment based on orthogonal projections are very similar. The differences lie in the information represented by the matrices $\mathbf{P}$. For the NAS, $\mathbf{P}$ contains the vectors 
spanning all the spectral contributions to be removed. In general pretreatment cases, $\mathbf{P}$ contains the vectors spanning only a part of the space containing the contributions to be removed. The extent to which pretreatments are able to gather the comprehensive information to be removed determines how close corrected spectra will be to their NAS. And from equation 9, determining regression vector $\mathbf{b}$ is straightforward. The relationship between the NAS and $\mathbf{b}$, along with the calculation of the NAS using Euclidian orthogonal projections, has at least two consequences.

A first consequence is the embedded / not embedded property of pretreatments. A pretreatment is embedded into the calibration if it is not necessary to apply the pretreatment to a new spectrum $\mathbf{x}_{i, v a l}$ before using it for prediction. If Euclidian orthogonal projection is applied, e.g. to correct the spectra from $\mathbf{P}$, the regression vector $\mathbf{b}$ of the subsequent calibration is built in a subspace already orthogonal to $\mathbf{P}: \mathbf{b}=\mathcal{P} \frac{\perp}{P} \mathbf{b}$. Thus, it is not necessary to apply the same pretreatment to $\mathbf{x}_{i, v a l}$. However this property is not verified for non-Euclidian orthogonal projections. For example, OSC methods have different behaviors. Fearn's OSC is not embedded because it is an oblique rather than an Euclidan projection. In contrast, the DO and NAP OSCs, which are written as Euclidian orthogonal projections, are embedded. No conclusions can be drawn for the other OSC methods because they have not yet been rewritten as projections into $\mathbb{R}^{P}$.

A second consequence is an identification of the useful and detrimental spaces within the variables space $\mathbb{R}^{P}$ (Fig.2). The useful space $\mathcal{E}^{U}$ contains informations for the prediction of the compound of interest. Its dimension is one according to the first definition, but can be greater than one according to the second definition. The subspace related to other compounds or chemical influences is detrimental $\left(\mathcal{E}^{D}\right)$ if it shares common information with the useful space. The information from $\mathcal{E}^{U}$ which is not shared by $\mathcal{E}^{D}$ is the NAS, i.e. the NAS is orthogonal to $\mathcal{E}^{D}$. All the information from $\mathbb{R}^{P}$ which does not belong to $\mathcal{E}^{U}$ or $\mathcal{E}^{D}$ is unuseful information because it is already orthogonal to $\mathcal{E}^{U}$. Pretreatments have different strategies to remove $\mathcal{E}^{D}$. OSC methods rely on the information provided by $\mathbf{y}$. However, as PLSR uses the same approach, there is no added value, at least for prediction. Nevertheless OSCs calculated using other data (e.g. pure spectra or experimental design) and then applied to the calibration data $\mathbf{X}$ can increase the accuracy of measurement $(36 ; 37)$ because the two sets of spectral informations are complementary. On the other hand, the non OSC orthogonal projections (OSP, IIR, EPO, TOP, DOP, EROS, detrend, CPSA, DWT-WPT) rely on additional information, and various sources of information can be withdrawn. The disadvantages of the methods are: (1) except for DWT-WPT, a prior knowledge is necessary, such as a model, pure spectra or information that can be used to build an experimental design; and (2) non OSC orthogonal pretreatments may remove information from the NAS subspace, contributing to a drop in the accuracy of prediction. Therefore, choosing the correct pretreatments and tuning and applying them properly, are very important for optimizing their predictive value. 


\subsection{Pretreatment complementarity}

A spectrum can be viewed as the sum of signals of different frequencies: low, medium, and high (38).

Baseline shifts correspond to low frequencies. They can be modeled and removed either by detrend and related methods or by data compression methods such as WPT. Detrend is used more often than WPT. One reason for this may be that the polynomials used are based on models of low rank (e.g. 2). These models may already fit well with the observed deviations of the baseline, and thus detrend is more parsimonious.

Noise corresponds to high frequencies, usually removed using the SG algorithm. However WPT also removes noise, smooths the spectra and is more compatible with the other orthogonal projections.

Most of the relevant spectral information corresponds to medium frequencies, such as the information from the component of interest and the information to be removed. Orthogonal projections perform best in these situations.

Therefore different pretreatments should be selected according to their complementarity; that is, their ability to represent the whole space to be removed (Fig. 2, hatched part). Pretreatment choice is guided by the data to be processed and also by the available information. However, disrupting information can be expected in all frequency ranges. Thus, a relevant association of several methods, such as detrend + EPO + WPT, would respectively be able to remove the low, medium and high frequency spectral perturbations. How can several projection methods be used to produce the best results?

\subsection{Association of pretreatments}

Using successively two or more orthogonal projections is not equivalent to performing a single orthogonal projection containing all the information to be removed. An example is given in Fig. 1. Suppose that the spectral information from water and ethanol are to be removed from the spectrum of a sample that is half ethanol and half water. The expected result is the null vector. It is obtained if the orthogonal projection to ethanol and water is done in one step, but not if two orthogonal projections, one for ethanol and one for water, are done separately. Moreover, successive orthogonal projections are not commutative, so different orders lead to different results.

Let $\mathbf{P}$ and $\mathbf{Q}$ be two matrices whose column-vectors span the subspaces $\mathcal{E}_{P}$ and $\mathcal{E}_{Q}$. Let $\mathcal{P}_{P}$ and $\mathcal{P}_{Q}$ be the orthogonal projectors for $\mathcal{E}_{P}$ and $\mathcal{E}_{Q}$, respectively. The combined orthogonal projector for the $\mathcal{E}_{P}$ and $\mathcal{E}_{Q}$ subspaces is called $\mathcal{P}_{P+Q}$. According to Piziak et al (39):

$$
\mathcal{P}_{P+Q}=\left(\mathcal{P}_{P}+\mathcal{P}_{Q}\right)\left(\mathcal{P}_{P}+\mathcal{P}_{Q}\right)^{+}
$$

The orthogonal projector to $\mathcal{E}_{P}$ and $\mathcal{E}_{Q}$ is $\mathcal{P} \frac{1}{P+Q}$ defined as:

$$
\mathcal{P}_{P}^{\perp}+Q=\mathbf{I}_{P}-\left(\mathcal{P}_{P}+\mathcal{P}_{Q}\right)\left(\mathcal{P}_{P}+\mathcal{P}_{Q}\right)^{+}
$$


If $\mathcal{E}_{P}$ and $\mathcal{E}_{Q}$ are orthogonal, equation 10 is simplified:

$$
\mathcal{P}_{P}^{\perp}+Q=\mathbf{I}_{P}-\mathcal{P}_{P}-\mathcal{P}_{Q}=\left(\mathbf{I}_{P}-\mathcal{P}_{P}\right)\left(\mathbf{I}_{P}-\mathcal{P}_{Q}\right)=\mathcal{P}_{P}^{\perp} \mathcal{P}_{Q}^{\perp}
$$

However, in the other cases where $\mathcal{E}_{P}$ and $\mathcal{E}_{Q}$ are not orthogonal, $\mathcal{P}_{P}^{\perp}+Q \neq$ $\mathcal{P} \perp \mathcal{P} \mathcal{P}_{Q}^{\perp}$. So, if several Euclidian orthogonal pretreatments must be applied, it is strongly recommended to merge their respective $\mathbf{P}$ matrices into a matrix $\mathbf{R}$ and then performing a single orthogonal projection to $\mathbf{R}$. For example, suppose that spectra are to be corrected from scattering and from chemical components whose pure spectra are known. The best choice is to merge the $\boldsymbol{\Lambda}_{2}$ to the pure spectra, yielding $\mathbf{R}$, and to orthogonalize to $\mathbf{R}$. This is also the option recommended by Brown (15). In the case of successive orthogonal projections, calculations will be erroneous if the bases of the different subspaces are not orthogonal.

\subsection{Limits of orthogonal projections}

Orthogonal projections have at least two limits: the number of dimensions of the spectra to be processed and the correction of multiplicative effects.

\subsubsection{Reduction of dimensions and number of variables}

Orthogonal projection to a subspace of dimension $A$ retains the number of variables but reduces the mathematical dimension of the spectra by $A$, which cannot exceed the dimension $P$ of the spectra. This is not a problem if spectra contain hundreds of variables, because $A$ remains lower than $P$. However, if the spectra contain only a few variables (e.g. between 10 and 20), then only a few dimensions can be removed by orthogonal projections, and often orthogonal projection cannot be applied.

\subsubsection{Additive and multiplicative effects}

Orthogonal projection methods correct additive effects according to equation 1. Suppose that the spectral perturbations also induce a multiplicative effect $\alpha_{i}$, such that Eq. 1 can be written:

$$
\mathbf{x}_{i, o b s}=\alpha_{i}\left(\mathbf{x}_{i}+\mathbf{h}_{i}\right)=\alpha_{i} \mathbf{x}_{i}+\alpha_{i} \mathbf{h}_{i}
$$

An orthogonal projector $\mathcal{P}_{R}^{\perp}$ can be obtained such that:

$$
\mathbf{x}_{i, c o r r}=\mathbf{x}_{i, o b s} \mathcal{P}_{R}^{\perp}=\alpha_{i} \mathbf{x}_{i} \mathcal{P}_{R}^{\perp}
$$

Orthogonal projections are able to remove the term $\alpha_{i} \mathbf{h}_{i}$, but are not able to correct the value $\alpha_{i}$ associated with $\mathbf{x}_{i}$; they cannot handle multiplicative effects. Thus, methods such as standard normal variate (SNV) proposed by Barnes (11), or (extended) multiplicative signal correction proposed by Martens $(40 ; 41)$ do not use orthogonal projections to correct multiplicative effects. 


\section{Conclusion}

All the pretreatments presented here are based on orthogonal projections. Some pretreatments that also use the principle of orthogonal projection were not adressed because they do not fulfill all the conditions. The MSC and EMSC methods perform a corrections using subtraction and division. SG is a piecewise orthogonal projection. Even variable selection is an orthogonal projection into the variables space, but in practice, variables are dropped by reshaping the spectra rather than by setting them to 0 . Thus, the orthogonal projection principle, which is directly associated with the least squares approximation, is used extensively and its prevalence is a confirmation of its power. Projections are performed usually in a Euclidian space but at least one OSC is an oblique projection performed in a non-Euclidian space.

The information used by calibrations is actually an estimation of the NAS. A very important issue for pretreatments is identifying the detrimental information to be removed by orthogonal projection. Two opposing strategies are used: supervised and unsupervised. On one hand, OSC methods are supervised, the information to be removed is chosen orthogonal to the reference values $\mathbf{y}$. These methods obey the definition of the NAS, and thus OSC methods should perform very well. However, due to OSC's redundancy with PLSR, which is based on the same principles its accuracy has not improved substantially. On the other hand, information removed by unsupervised orthogonal projection methods is chosen based on prior knowledge of the experimental conditions and the analyzed samples. All the available information, pure spectra, spectra from an experimental design, and models (such as polynomials), should be considered to correct the detrimental information as comprehensively as possible. It is important to avoid removing any information about the compound of interest, which would lead to worse calibration models. Thus, proper use of orthogonal projections requires being very careful about the quality and the completeness of the removed information.

Orthogonal projections have practical properties. Information from different origins should be merged in order to process only one orthogonal projection. Euclidian orthogonal projections are embedded; they are applied only once when building the calibration model. There is no need to apply them to new spectra. Orthogonal projections also have limits. They cannot be applied in good conditions for spectra containing just a few variables, and they do not correct multiplicative effects. Nevertheless, orthogonal projections have proven to be valuable tools when building the calibration models of many applications.

\section{Acknowledgements}

The authors would like to thank the reviewers whose relevant remarks were very helpful for the improvement of this paper. 


\begin{tabular}{|c|c|}
\hline $\mathbf{X}$ & matrix $N \times P, N$ samples and $P$ spectral variables \\
\hline $\mathbf{y}$ & vector $N \times 1$, the reference values \\
\hline $\mathbf{X}_{1: i}$ & projection of $\mathbf{X}$ orthogonaly to $\left\{\mathbf{t}_{1}, \mathbf{t}_{2}, \ldots \mathbf{t}_{i}\right\}$ \\
\hline $\mathbf{T}$ & matrix $N \times A$, scores for $\mathbf{X}$ \\
\hline $\mathbf{P}$ & matrix $P \times A$, loadings for $\mathbf{X}$ \\
\hline $\mathbf{W}$ & Moore-Penrose pseudo-inverse of $\left(\mathbf{X}^{\prime} \mathbf{X}\right) ; \mathbf{\Sigma}=\left(\mathbf{X}^{\prime} \mathbf{X}\right)^{+}$ \\
\hline $\boldsymbol{\Sigma}$ & identity matrices for $\mathbb{R}^{N}$ and $\mathbb{R}^{P}$ spaces \\
\hline $\mathbf{I}_{N}, \mathbf{I}_{P}$ & Euclidian orthogonal projector $P \times P$ onto $\mathbf{P} ; \mathcal{P}_{P}=\mathbf{P}\left(\mathbf{P}^{\prime} \mathbf{P}\right)^{-1} \mathbf{P}^{\prime}$ \\
\hline $\mathcal{P}_{P}$ & $i^{\text {eme }}$ column vector of $\mathbf{T}$ \\
\hline $\mathcal{P}_{P}^{\perp}$ & $i^{\text {eme }}$ column vector of $\mathbf{P}$ \\
\hline $\mathbf{t}_{i}$ & $i^{\text {eme }}$ column vector of $\mathbf{W}$ \\
\hline $\mathbf{p}_{i}$ & subspace of $\mathbb{R}^{P}$ spanned by the line vectors of $\mathbf{X}$ \\
\hline $\mathbf{w}_{i}$ & useful $/$ detrimental subspaces of $\mathbb{P}^{P}$ \\
\hline $\mathcal{E}_{X}$ & \\
\hline $\mathcal{E}^{U}, \mathcal{E}^{D}$ & \\
\hline
\end{tabular}

Table 1: Main notations 


\begin{tabular}{|c|c|}
\hline$\overline{\mathrm{BB}}$ & best basis \\
\hline CPSA & constrained principal spectra analysis \\
\hline $\mathrm{DFT}$ & discrete Fourier transform \\
\hline $\mathrm{DO}$ & direct orthogonalization \\
\hline DOP & dynamic orthogonal projection \\
\hline DOSC & direct orthogonal signal correction \\
\hline DWT & discrete wavelet transform \\
\hline EMSC & extended multiplicative signal correction \\
\hline $\mathrm{EPO}$ & external parameter orthogonalization \\
\hline EROS & error removal by orthogonal subtraction \\
\hline IIR & independent interference reduction \\
\hline MSC & multiplicative signal correction \\
\hline MDL & minimum description length \\
\hline NAP & net analyte preprocessing \\
\hline NAS & net analyte signal \\
\hline NSV & net sensitivity vector \\
\hline OPLS & orthogonal projection to latent structures \\
\hline $\mathrm{OSC}$ & orthogonal signal correction \\
\hline $\mathrm{OSP}$ & orthogonal subspace projection \\
\hline $\mathrm{PCA}$ & principal component analysis \\
\hline PCDA & principal component discriminant analysis \\
\hline PLSR & projection to latent structures regression \\
\hline RMSEP & root mean square error of prediction \\
\hline SG & Savitsky-Golay \\
\hline SNV & standard normal variate \\
\hline SVD & singular value decomposition \\
\hline TOP & transfer by orthogonal projection \\
\hline WPT & wavelet packet transform \\
\hline
\end{tabular}

Table 2: Glossary 


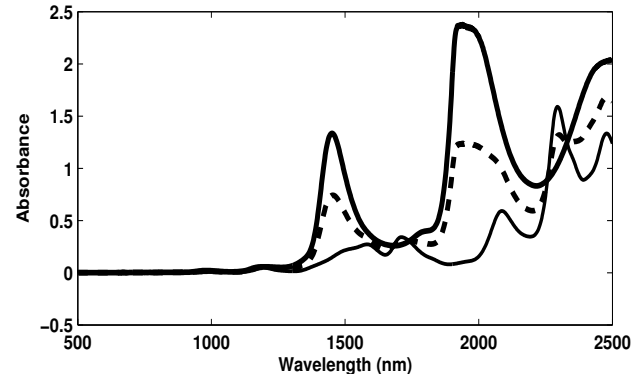

(1)

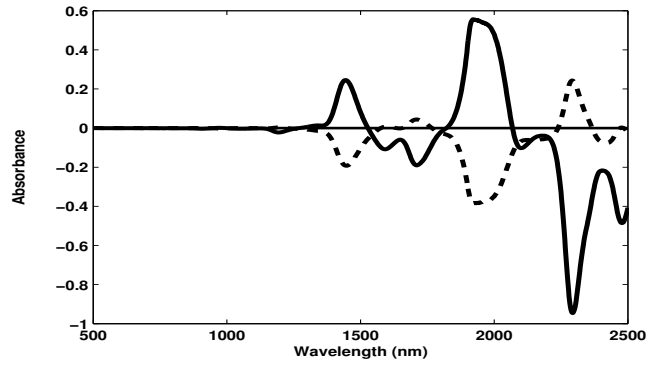

(2)

Figure 1: Water and ethanol spectra, and their orthogonal projection. (1) From top to bottom, spectra of water, water+ethanol, ethanol. (2) Projection of the (water+ethanol) spectrum orthogonally to: ethanol then water (thick line), water then ethanol (dashed line), and a matrix containing water and ethanol spectra (horizontal light line)

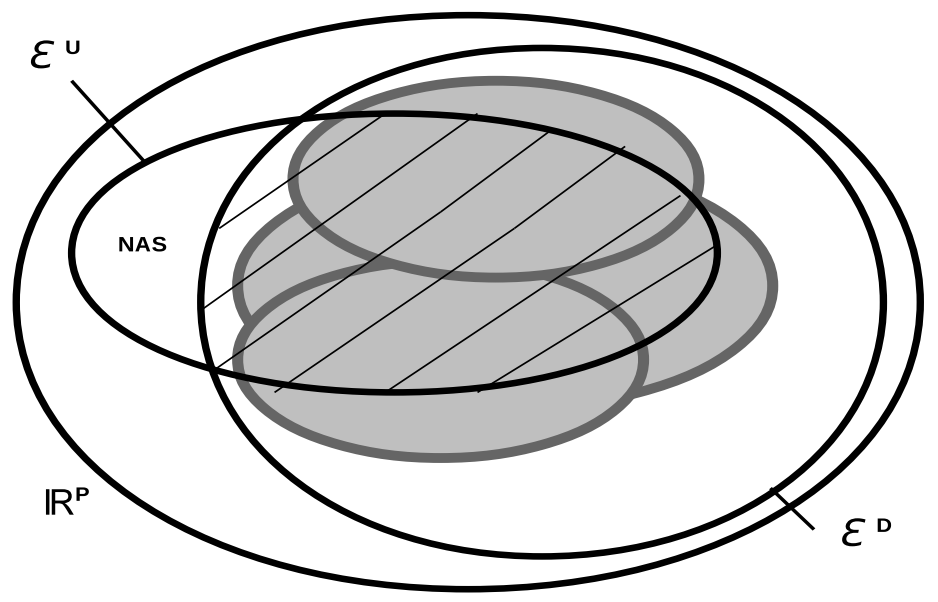

Figure 2: Different subspaces of $\mathbb{R}^{P}$. The useful subspace $\mathcal{E}^{U}$; the detrimental subspace $\mathcal{E}^{D}$; the NAS; the common part of $\mathcal{E}^{U}$ and $\mathcal{E}^{D}$ to be removed (hatched); and the information represented by three orthogonal pretreatments (grey) are depicted in the figure. Information inside of and outside of each subspace are orthogonal 


\section{References}

[1] S.Wold, A.Ruhe, H.Wold, W. D. III, The collinearity problem in linear regression, the partial least square (pls) approch to generalized inverses, J. Sci Stat. Comput. 5 (1984) 735-743.

[2] A.Rinnan, F.VanDenBerg, S. Engelsen, Review of the most common preprocessing techniques for near infrared spectra, TrAC 28(10) (2009) 12011222 .

[3] C.I.Chang, M.L.G.Althouse, Unsupervised interference rejection approach to target detection and classification for hyperspectral imagery, Opt. Eng. 37(3) (1998) 735-743.

[4] C.I.Chang, Hyperspectral imaging, Kluver academic / Plenum, N.Y., 2003.

[5] J.C.Harsanyi, C.I.Chang, Hyperspectral image classification and dimensionality reduction: an orthogonal subspace projection approach, IEEE Trans. Geosci. Rem. Sens. 32(4) (1994) 779-786.

[6] P.W.Hansen, Pre-processing method minimizing the need for reference analyses, J. Chemom. 15 (2001) 123-131.

[7] J.M.Roger, F.Chauchard, V.Bellon-Maurel, Epo-pls external parameter orthogonalisation of pls, application to temperature-independant measurement of sugar contents in fruits, Chemom. Intell. Lab. Syst. 66 (2003) 191-204.

[8] A.Andrew, T.Fearn, Transfer by orthogonal projection: making near infrared calibrations robust to between-instrument variation, Chemom. Intell. Lab. Syst. 72 (2004) 51-56.

[9] M.Zeaiter, J.M.Roger, V.Bellon-Maurel, Dynamic orthogonal projection, a new method to maintain the on-line robustness of multivariate calibration, application to nir-based monitoring of wine fermentations., Chemom. Intell. Lab. Syst. 80 (2006) 227-235.

[10] Y.Zhu, T.Fearn, D.Samuel, A.Dhar, O.Hameed, S.G.Brown, L.B.Lovat, Error removal by orthogonal substraction (eros): a customised pre-treatment for spectroscopic data, J. Chemom. 22 (2008) 130-134.

[11] R.J.Barnes, M.S.Dhanoa, S.J.Lister, Standard normal variate transformation and de-trening of near-infrared diffuse reflectance spectra, Appl. Spectrosc. 43 (1989) 772-777.

[12] R.J.Barnes, M.S.Dhanoa, S.J.Lister, Correction to the description of standard normal variate (snv) and de-trend (dt) transformations in practical spectroscopy with applications in food and beverage analysis, 2nd edition, J. Near Infrared Spectrosc. 1 (1993) 185-186. 
[13] V.Mazet, C.Carteret, D.Brie, J.Idier, B.Humbert, Background removal from spectra by designing and minimizing a non-quadratic cost function, Chemom. Intell. Lab. Syst. 76 (2005) 121-133.

[14] F.Gan, G.Ruan, J.Mo, Baseline correction by improved iterative polynomial fitting with automatic threshold, Chemom. Intell. Lab. Syst. 82 (2006) $59-65$.

[15] J.M.Brown, Method for correcting spectral data for data due to the spectral measurement process itself and estimating unknown property and/or composition data of a sample using such method, U.S. patent 5.121.337 (1992).

[16] B.Walczak, D.L.Massart, Noise suppression and signal compression using the wavelet packet transform, Chemom. Intell. Lab. Syst. 36 (1997) 81-94.

[17] J.Trygg, S.Wold, Pls regression on wavelet compressed nir spectra, Chemom. Intell. Lab. Syst. 42 (1998) 209-220.

[18] L.Eriksson, J.Trygg, E.Johansson, R.Bro, S.Wold, Orthogonal signal correction, wavelet analysis and multivariate calibration of complicated process fluorescence data, Anal.Chim. Acta 420 (2000) 181-195.

[19] L.Pasti, B.Walczak, D.L.Massart, P.Reschiglian, Optimization of signal denoising in discrete wavelet transform, Chemom. Intell. Lab. Syst. 48 (1999) $21-34$.

[20] Y.Hu, T.Jiang, A.Shen, W.Li, X.Wang, J.Hu, A background elimination method based on wavelet transform for raman spectra, Chemom. Intell. Lab. Syst. 85 (2007) 94-101.

[21] R.K.H.Galvao, G.E.Jose, H.A.D.Filho, M.C.V.Aranjo, E.C.DaSilva, H.M.Paiva, T.C.B.Saldanha, E.S.O.N.DeSouza, Optimal wavelet filter construction using x and y data, Chemom. Intell. Lab. Syst. 70 (2004) 1-10.

[22] B.K.Alsberg, A.M.Woodward, D.B.Bell, An introduction to wavelet transform for chemometricians: a time-frequency approach, Chemom. Intell. Lab. Syst. 37 (1997) 215-239.

[23] V.J.Barclay, R.F.Bonner, I.P.Hamilton, Application of wavelet transforms to experimental spectra: smoothing, denoising and data compression, Anal. Chem. 69 (1997) 78-90.

[24] S. Wold, H. Antti, F. Lindgren, J. Ohman, Orthogonal signal correction of near infra-red spectra., Chemom. Intell. Lab. Syst. 44 (1998) 175-185.

[25] J.Sjoblom, O.Svensson, M.Josefson, H.Kullberg, S.Wold, An evaluation of orthogonal signal correction applied to calibration transfer of near infrared spectra, Chemom. Intell. Lab. Syst. 44 (1998) 229-244. 
[26] J.A.Westerhuis, J.DeJong, A.K.Smilde, Direct orthogonalization, Chemom. Intell. Lab. Syst. 56 (2001) 13-25.

[27] C.A.Andersson, Direct orthogonalization, Chemom. Intell. Lab. Syst. 47 (1999) 51-63.

[28] T.Fearn, On orthogonal signal correction., Chemom. Intell. Lab. Syst. 50 (2000) 47-52.

[29] H.C.Goicoechea, A.C.Olivieri, A comparison of orthogonal signal correction and net analyte preprocessing methods, theorical and experimental study, Chemom. Intell. Lab. Syst. 56 (2001) 73-81.

[30] J.Trygg, S.Wold, Orthogonal projection to latent structures (o-pls), J. of Chemom. 16(3) (2002) 119-128.

[31] O.Svensson, T.Kourti, J.F.MacGregor, An investigation of orthogonal signal correction algorithms and their characteristics, J. of Chemom. 16(4) (2002) 176-188.

[32] E.K.Kemsley, H.S.Tapp, Opls filtered data can be obtained directly from non-orthogonalized pls1, J. Chemom. 23 (2009) 518-529.

[33] A.Lorber, N.K.M.Faber, B.R.Kowalski, Net analyte signal calculation in multivariate calibration, Anal. Chem. 69(8) (1997) 1620-1626.

[34] J.Ferre, N.K.M.Faber, Net analyte signal calculation for multivariate calibration, Chemom. Intell. Lab. Syst. 69 (2003) 123-136.

[35] R.Bro, C.M.Andersen, Theory of net analyze signal vectors in inverse regression, J. of Chemom. 17 (2003) 646-652.

[36] J.C.Boulet, T.Doco, J.M.Roger, Improvement of calibration models using two successive orthogonal projection methods, application to quantification of wine mannoproteins., Chemom. Intell. Lab. Syst. 87 (2007) 295-302.

[37] S.Preys, J.M.Roger, J.C.Boulet, Robust calibration using orthogonal projection and experimental design, application to the correction of the light scattering effect on turbid nir spectra., Chemom. Intell. Lab. Syst. 91 (2006) $28-33$.

[38] K.H.Liland, T.Almoy, B.H.Mevik, Optimal choice of baseline correction for multivariate calibration of spectra, Appl. Spectrosc. 64(9) (2010) 10071016 .

[39] R.Piziak, P.L.Odell, R.Hahn, Constructing projections on sums and intersections, Comput. Math. Appl. 37 (1999) 67-74.

[40] P.Geladi, D.MacDougall, H.Martens, Linearization and scatter-correction for near infrared reflectance spectra of meat, Appl. Spectrosc. 39(3) (1985) 491-499. 
[41] H.Martens, E.Stark, Extended multiplicative signal correction and spectral interference substraction: new preprocessing methods for near infrared spectroscopy, J. Pharm. Biomed. Anal. 9 (1991) 625-635.

[42] R.DeMaesschlack, D.JouanRimbaud, D.L.Massart, The mahalanobis distance, Chemom. Intell. Lab. Syst. 50 (2000) 1-18.

\section{Appendix A. Writing Fearn's OSC in the form of an orthogonal pro- jection into $\mathbb{R}^{P}$}

Two equations are extracted from Fearn's text:

$$
\begin{aligned}
\mathbf{t}_{i} & =\mathbf{X} \mathbf{w}_{i} \\
\mathbf{p}_{i} & =\mathbf{X}^{\prime} \mathbf{t}_{i}\left(\mathbf{t}^{\prime}{ }_{i} \mathbf{t}_{i}\right)^{-1}
\end{aligned}
$$

Let $\boldsymbol{\Sigma}$ be $\left(\mathbf{X}^{\prime} \mathbf{X}\right)^{+}$, the Moore-Penrose pseudo-inverse of $\left(\mathbf{X}^{\prime} \mathbf{X}\right)$. Our first goal is to write $\mathbf{w}_{i}$ using $\mathbf{p}_{i}$ and $\boldsymbol{\Sigma}$. The calculation of the product $\mathbf{p}_{i}^{\prime}{ }_{i} \boldsymbol{\Sigma} \mathbf{p}_{j}$ is performed using equations A.1 and A.2 :

$$
\begin{aligned}
\mathbf{p}_{i}^{\prime}{ }_{i} \boldsymbol{\Sigma} \mathbf{p}_{j} & =\left(\mathbf{t}^{\prime}{ }_{i} \mathbf{t}_{i}\right)^{-1} \mathbf{w}_{i}{ }_{i} \mathbf{X}^{\prime} \mathbf{X} \boldsymbol{\Sigma} \mathbf{X}^{\prime} \mathbf{X} \mathbf{w}_{j}\left(\mathbf{t}^{\prime}{ }_{j} \mathbf{t}_{j}\right)^{-1} \\
& =\left(\mathbf{t}^{\prime}{ }_{i} \mathbf{t}_{i}\right)^{-1} \mathbf{w}_{i}{ }_{i} \mathbf{X}^{\prime} \mathbf{X} \mathbf{w}_{j}\left(\mathbf{t}^{\prime}{ }_{j} \mathbf{t}_{j}\right)^{-1} \\
& =\left(\mathbf{t}^{\prime}{ }_{i} \mathbf{t}_{i}\right)^{-1} \mathbf{t}^{\prime}{ }_{i} \mathbf{t}_{j}\left(\mathbf{t}^{\prime}{ }_{j} \mathbf{t}_{j}\right)^{-1}
\end{aligned}
$$

If $i \neq j$, because of the orthogonality of the $\mathbf{t}_{i}$ :

$$
\begin{aligned}
\mathbf{p}_{i}^{\prime} \boldsymbol{\Sigma} \mathbf{p}_{i} & =\left(\mathbf{t}_{i}^{\prime} \mathbf{t}_{i}\right)^{-1} \\
\mathbf{p}_{i}^{\prime} \boldsymbol{\Sigma} \mathbf{p}_{j} & =0
\end{aligned}
$$

After multiplying on the left by $\boldsymbol{\Sigma} \mathbf{X}^{\prime}$, a different combination of equations A.1 and A.2, completed with equation A.3, yields:

$$
\boldsymbol{\Sigma} \mathbf{X}^{\prime} \mathbf{X} \mathbf{w}_{i}=\boldsymbol{\Sigma} \mathbf{X}^{\prime} \mathbf{t}_{i}=\boldsymbol{\Sigma} \mathbf{p}_{i}\left(\mathbf{t}_{i}^{\prime} \mathbf{t}_{i}\right)=\boldsymbol{\Sigma} \mathbf{p}_{i}\left(\mathbf{p}_{i}^{\prime} \boldsymbol{\Sigma} \mathbf{p}_{i}\right)^{-1}
$$

The left term is the projection of $\mathbf{w}_{i}$ onto the space spanned by $\mathbf{X}^{\prime} \mathbf{X}$, which is also the space spanned by the lines of $\mathbf{X}(39)$, called $\mathcal{E}_{X}$. On the other hand, the $\mathbf{w}_{i}$ are the eigenvectors of the matrix $\mathbf{M} \mathbf{X}^{\prime} \mathbf{X}$, where $\mathbf{M}$ is the projector orthogonal to $\mathbf{X}^{\prime} \mathbf{y}$, which belongs to $\mathcal{E}_{X}$. Thus, $\mathbf{w}_{i}$ also belongs to $\mathcal{E}_{X}$, it is not modified after projection onto $\mathbf{X}^{\prime} \mathbf{X}$ and equation A.5 is simplified:

$$
\mathbf{w}_{i}=\boldsymbol{\Sigma} \mathbf{p}_{i}\left(\mathbf{p}_{i}^{\prime} \mathbf{\Sigma} \mathbf{p}_{i}\right)^{-1}
$$


${ }_{647}$ The $\mathbf{p}_{i}$ are $\boldsymbol{\Sigma}$ - orthogonal according to equation A.4, and therefore $\left(\mathbf{P}^{\prime} \boldsymbol{\Sigma} \mathbf{P}\right)$ ${ }_{648}$ is a diagonal matrix of full rank. Thus, equation A.6 can be written in a matrix 649 form:

$$
\mathbf{W}=\boldsymbol{\Sigma} \mathbf{P}\left(\mathbf{P}^{\prime} \boldsymbol{\Sigma} \mathbf{P}\right)^{-1}
$$

The OSC correction is:

$$
\begin{aligned}
\mathbf{X}_{O S C} & =\mathbf{X}-\mathbf{X} \sum_{i=1}^{A} \mathbf{w}_{i} \mathbf{p}_{i}^{\prime} \\
& =\mathbf{X}-\mathbf{X} \mathbf{W} \mathbf{P}^{\prime} \\
& =\mathbf{X}\left(\mathbf{I}_{P}-\boldsymbol{\Sigma} \mathbf{P}\left(\mathbf{P}^{\prime} \boldsymbol{\Sigma} \mathbf{P}\right)^{-1} \mathbf{P}^{\prime}\right)
\end{aligned}
$$

${ }_{651}$ To conclude, Fearn's OSC can be also expressed into $\mathbb{R}^{P}$ as an oblique (not ${ }_{652}$ Euclidian) projection to the space spanned by the column-vectors of $\mathbf{P}$. If $\mathbf{X}$ ${ }_{653}$ has been centered, $\boldsymbol{\Sigma}$ is a metric associated to a Mahalanobis distance (42). 